\title{
Resonance enhancement of $x$ rays in layered materials: Application to surface enrichment in polymer blends
}

\author{
B. N. Dev* and Amal K. Das \\ Institute of Physics, Sachivalaya Marg, Bhubaneswar 751 005, India \\ S. Dev ${ }^{\dagger}$ \\ European Molecular Biology Laboratory Hamburg Outstation, c/o Deutsches Elektronen-Synchrotron, Notkestrasse 85, 22603 \\ Hamburg, Germany \\ D. W. Schubert \\ GKSS Forschungszentrum, Max-Plank-Strasse, 21502 Geesthacht, Germany \\ M. Stamm \\ Institut für Polymerforschung Dresden e.V., Hohè Strasse 6, 01069 Dresden, Germany \\ G. Materlik \\ Hamburgersynchrotronstrahlungslabor HASYLAB at Deutsches Elektronen-Synchrotron, Notkestrasse 85, 22603 Hamburg, Germany
}

(Received 21 October 1999)

\begin{abstract}
Resonance enhancement of $\mathrm{x}$ rays of both odd and even orders has been observed in a thin polymer blend film of polystyrene (PS) and polybromostyrene $\left(\mathrm{PBr}_{x} \mathrm{~S}\right)$ spin cast on a smooth Au layer on a silicon substrate. The X-ray intensity enhancement has been measured by detecting fluorescence from the $\mathrm{Br}$ atoms in the $\mathrm{PBr}_{x} \mathrm{~S}$ component of the compatible polymer blend. Analysis of the $\mathrm{Br} K \alpha$ fluorescence has yielded the $\mathrm{PBr}_{x} \mathrm{~S}$ distribution in the polymer blend layer in agreement with a PS surface enrichment model. PS is largely enriched at the free surface of the polymer layer and partially enriched at the underlying interface.
\end{abstract}

\section{INTRODUCTION}

A variety of physical phenomena occurs when $\mathrm{x}$ rays are incident at glancing angles on flat surfaces of materials. These phenomena include total external reflection, ${ }^{1}$ interference fringes in reflection from layers on a substrate, ${ }^{2}$ and the formation of evanescent ${ }^{3,4}$ and standing waves. ${ }^{5}$ All these phenomena have been utilized for the characterizations of surfaces, interfaces, and thin films. For thin-film preparation, among other techniques, spin coating is widely used in the semiconductor industry. ${ }^{6,7}$ Spin-coated polymer layers on solid substrates have been extensively studied by $\mathrm{x}$-ray reflectometry. ${ }^{8,9}$ Here we demonstrate the resonance enhancement of $\mathrm{x}$-ray intensity in such layers and describe its usefulness for the study of polymer layers and other layered materials in general.

Resonance enhancement effects have been observed under glancing incidence conditions in thin films on a substrate. These are of two different varieties - one below the critical angle of the top layer in a thin-film waveguide structure ${ }^{10}$ and the other above the critical angle. ${ }^{11,12}$ Wang et al. ${ }^{11}$ observed the resonance enhancement effect in a lipid multilayer film, prepared by the Langmuir-Blodgett (LB) technique, incorporating a heavy atom layer approximately at the middle of the LB layer during preparation. They observed only odd order enhancement peaks. Here we report on our observations of both odd and even order resonance enhancement in a polymer-blend layer, prepared by the usual spin coating on a gold-coated silicon substrate. The polymer-blend layer consists of polystyrene (PS) and polybromostyrene $\left(\mathrm{PBr}_{x} \mathrm{~S}\right)$. We use the notation $\mathrm{PBr}_{x} \mathrm{~S}$ for a statistical copolymer of poly ( $p$-bromostyrene-stat-styrene), which consists of statistically alternating monomers of para-bromostyrene at molar concentration $x$ and of styrene at molar concentration $(1-x)$. The experiment involves $\mathrm{x}$-ray reflectivity measurement with the simultaneous detection of $\mathrm{Br} K \alpha$ fluorescent $\mathrm{x}$ rays. Both odd- and even-order enhancements are manifested as peaks in the $\mathrm{Br} K \alpha$ fluorescence yield. Analysis of the fluorescence yield has been utilized to extract the depth distribution of $\mathrm{PBr}_{0.06} \mathrm{~S}$, which shows a surface and interface enrichment of the PS component of the polymer blend. This distribution could not be determined from standard x-ray reflectivity measurements because of the negligible electron density difference between the two components in the polymer blend.

\section{THEORY}

We give a brief theoretical background for the resonance enhancement process. We mainly follow the formalisms given by Parratt ${ }^{13}$ and de Boer ${ }^{12}$ for the reflectivity and then give the expression for the field intensity.

\section{A. Reflection from a multilayer system}

If all interfaces are parallel in a multilayer system (Fig. 1), a plane electromagnetic wave of frequency $\omega$ in a medium $j$ at a position $\mathbf{r}$ can be written as

$$
E_{j}(\mathbf{r})=E_{j}(0) \exp \left[i\left(\omega t-\mathbf{k}_{j} \cdot \mathbf{r}\right)\right]
$$

where $E_{j}(0)$ is the field amplitude at the top of the $j$ th layer. 


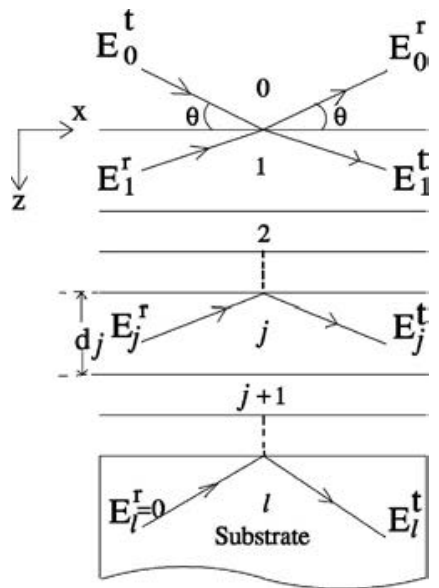

FIG. 1. A schematic representation of x-ray reflection from a multilayer system. See text for details.

For all $j$, the components of the wave vector, $\mathbf{k}_{j}=\mathbf{k}_{j}^{\prime}$ $-i \mathbf{k}_{j}^{\prime \prime}$, are given by

$$
k_{j, x}=\frac{2 \pi}{\lambda} \cos \theta, \quad k_{j, z}=\frac{2 \pi}{\lambda}\left(\epsilon_{j}-\cos ^{2} \theta\right)^{1 / 2},
$$

where $\theta$ is the glancing angle of incidence, $\lambda$ is the wavelength of the incident $\mathrm{x}$ rays, and the dielectric function $\epsilon_{j}$ is given by

$$
\epsilon_{j}=1-2 \delta_{j}-i 2 \beta_{j}
$$

where

$$
\delta=\left(\lambda^{2} / 2 \pi\right) r_{e} \rho_{e}, \quad \beta=(\lambda / 4 \pi) \mu .
$$

$\delta$ and $\beta$ are related to the real and imaginary parts of the atomic scattering factors, $\rho_{e}$ is the electron density, and $\mu$ is the linear absorption coefficient for the incident photons in the medium. $r_{e}$ is the classical electron radius. We consider the medium for the incident beam to be vacuum with $\epsilon_{0}$ $=1$.

For the $s$ polarization of the electric field and smooth interfaces the complex coefficient of reflection $r_{j}$ and transmission $t_{j}$, being the ratio of electric fields at the $j, j+1$ interface, are given by Fresnel's formulas

$$
\begin{aligned}
& r_{j}=\frac{k_{j, z}-k_{j+1, z}}{k_{j, z}+k_{j+1, z}}, \\
& t_{j}=\frac{2 k_{j, z}}{k_{j, z}+k_{j+1, z}} .
\end{aligned}
$$

For rough interfaces these expressions are to be modified. There are several methods for obtaining modified expressions. In a well-known method ${ }^{14,15} r_{j}$ is multiplied by a factor $S_{j}$ given by

$$
S_{j}=\exp \left[-2 \sigma_{j}^{2} k_{j, z} k_{j+1, z}\right],
$$

where $\sigma_{j}$ is the root-mean-square deviation of the interface atoms from the perfectly smooth condition. An expression like Eq. (7) is only valid for small roughnesses $\left(\sigma_{j}\left|k_{j, z}\right|<1\right)$.

For the modification of $t_{j}$, it is to be multiplied by

$$
T_{j}=\exp \left[\sigma_{j}^{2}\left(k_{j, z}-k_{j+1, z}\right)^{2} / 2\right] .
$$

So far we have discussed the reflection and refraction at a single interface. For a multilayer system, involving multiple interfaces, the electric fields at all the interfaces can be obtained from either a recursion relation or from a matrix formalism. In the following we will use the recursion relation which was first introduced by Parratt. ${ }^{13}$ In the recursion method, ${ }^{12,13}$ the transmitted field $E_{j}^{t}$ and the reflected field $E_{j}^{r}$ at the top of the $j$ th layer are found from the relations

$$
\begin{gathered}
E_{j}^{r}=a_{j}^{2} X_{j} E_{j}^{t}, \\
E_{j+1}^{t}=\frac{a_{j} E_{j}^{t} t_{j} T_{j}}{1+a_{j+1}^{2} X_{j+1} r_{j} S_{j}},
\end{gathered}
$$

and

$$
X_{j}=\frac{\left(r_{j} S_{j}+a_{j+1}^{2} X_{j+1}\right)}{1+a_{j+1}^{2} X_{j+1} r_{j} S_{j}},
$$

where

$$
a_{j}=\exp \left(-i k_{j, z} d_{j}\right)
$$

$d_{j}$ being the thickness of the $j$ th layer.

For the substrate $E_{l}^{r}=X_{l}=0$. The electric-field amplitudes $E_{j}^{t}$ (transmitted) and $E_{j}^{r}$ (reflected) can be computed from the knowledge of $\lambda, \theta, \epsilon_{j}$ 's, the thickness of the layers $\left(d_{j}\right.$ 's), and the interface roughness $\left(\sigma_{j}\right.$ 's) using Eqs. (2) $-(12)$ and the reflectivity $R$ is then obtained from the ratio of $E$ fields outside the surface

$$
R(\theta)=\left|E_{0}^{r} / E_{0}^{t}\right|^{2}
$$

The interference between the incident $E$ field $\left(E_{j}^{t}\right)$ and the reflected $E$ field $\left(E_{j}^{r}\right)$ can form standing-waves within any layer and that between $E_{0}^{t}$ and $E_{0}^{r}$ can form standingwaves above the surface in vacuum. In order to obtain this standing-wave field in the $j$ th layer one needs to know the fields $E_{j}^{t}$ and $E_{j}^{r}$ as a function of depth $(z)$.

\section{B. Field intensity}

The total $E$ field at a point $\mathbf{r}$ in the $j$ th layer is given by

$$
\boldsymbol{E}_{j}^{T}(\mathbf{r})=\boldsymbol{E}_{j}^{t}(\mathbf{r})+\boldsymbol{E}_{j}^{r}(\mathbf{r}),
$$

where

$$
E_{j}^{t}(\mathbf{r})=E_{j}^{t}(0) \exp \left(-i k_{j, z} z\right) \exp \left[i\left(\omega t-k_{j, x} x\right)\right]
$$

and

$$
E_{j}^{r}(\mathbf{r})=E_{j}^{r}(0) \exp \left(+i k_{j, z} z\right) \exp \left[i\left(\omega t-k_{j, x} x\right)\right] .
$$

Here the origin has been chosen to be on the interface at the top of the $j$ th layer. Thus $E_{j}^{t}(0)$ and $E_{j}^{r}(0)$ represent the transmitted and the reflected $E$ fields at the top of the $j$ th layer. $E_{j}^{t}(0)$ and $E_{j}^{r}(0)$ are readily obtained from the recursion relations [Eqs. (9)-(12)]. ${ }^{12,13}$ The field intensity $I(\theta, z)$ $=\left|\boldsymbol{E}_{j}^{T}(\mathbf{r})\right|^{2}$ can be shown to be given by (for $s$ polarization) 


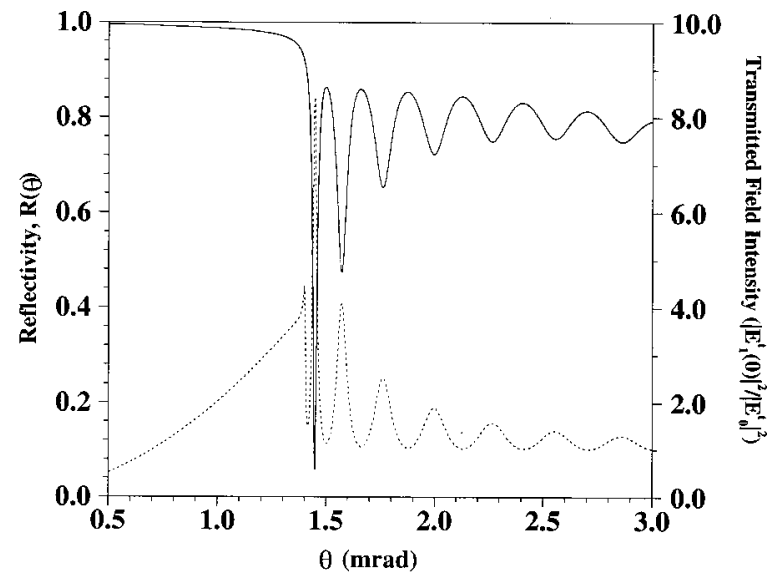

FIG. 2. Reflectivity (- $(-)$ and the transmitted field intensity $(\cdots \cdots)$ as a function of the angle of incidence [polymer blend $(1000 \AA) / \mathrm{Au}(500 \AA) / \mathrm{Si}]$.

$$
\begin{aligned}
I(\theta, z)= & \left|E_{j}^{t}(0)\right|^{2}\left[\exp \left\{-2 k_{j, z}^{\prime \prime} z\right\}+\left|\frac{E_{j}^{r}(0)}{E_{j}^{t}(0)}\right|^{2} \exp \left\{2 k_{j, z}^{\prime \prime} z\right\}\right. \\
& \left.+2\left|\frac{E_{j}^{r}(0)}{E_{j}^{t}(0)}\right| \cos \left(\nu+2 k_{j, z}^{\prime} z\right)\right]
\end{aligned}
$$

where $\nu$ is defined by $E_{j}^{r}(0) / E_{j}^{t}(0)=\left|E_{j}^{r}(0) / E_{j}^{t}(0)\right| e^{i \nu}$, i.e., $\nu$ is the phase of the $E$-field ratio at the top of the $j$ th layer. It is clear from Eq. (17) that a standing wave is generated within the $j$ th layer. If the absorption in the medium is ignored (i.e., $k_{j, z}^{\prime \prime}=0$ ), the quantity within the square brackets may attain a maximum value of 4 . However, the prefactor $\left|E_{j}^{t}(0)\right|^{2}$ is an oscillatory function of $\theta$ and may be quite large in comparison to the incident intensity $\left|E_{0}^{t}\right|^{2}$ under appropriate conditions giving rise to a large enhancement in $I(\theta, z)$.

The ratio of the transmitted intensity at the top of layer " 1 " (i.e., just below the surface) to the incident intensity $\left(\left|E_{1}^{t}(0) / E_{0}^{t}(0)\right|^{2}\right)$ is obtained from

$$
\left|E_{1}^{t}(0)\right|^{2}=\left|\frac{a_{0} t_{0}}{1+a_{1}^{2} X_{1} r_{0}}\right|^{2}\left|E_{0}^{t}\right|^{2} .
$$

This ratio is shown as a function of angle of incidence for a given system in Fig. 2 to elucidate the enhancement phenomenon. Details about the plots in Fig. 2 are discussed in Sec. II C.

\section{Examples of calculation}

In this section we present the results of calculations of various quantities in Secs. II A and II B using an example. We take a three-layer system: polymer-blend (1000 $\AA$ )/ $\mathrm{Au}(500 \AA) / \mathrm{Si}$ (substrate). In order to demonstrate the resonance enhancement effects of both odd and even orders, we will use an example of a polymer-blend film containing 15\% polystyrene (PS) and $85 \%$ polybromostyrene $\left(\mathrm{PBr}_{x} \mathrm{~S}\right.$ with $x$ $=0.06$ ) on a layer of higher electron density (here gold). The blend of $\mathrm{PS}$ with $\mathrm{PBr}_{x} \mathrm{~S}$ is also interesting from the viewpoint of polymer science. ${ }^{16,17}$ The properties of the $\mathrm{PS}^{-\mathrm{PBr}_{x} \mathrm{~S}}$ blend systems change with the degree of bromination $x$, giv- ing mixtures with various degrees of miscibility, segregation, and polymer interdiffusion. We take the trilayer system $\left[\mathrm{PS}(15 \%)+\mathrm{PBr}_{0.06} \mathrm{~S}(85 \%)\right](1000 \AA) / \mathrm{Au}(500 \AA) / \mathrm{Si}$. In this computation we have used the following parameters: $E_{\gamma}$ (energy of the incident $\mathrm{x}$ rays $)=16.9 \mathrm{keV}, \epsilon_{1}=1-(1.87$ $\left.\times 10^{-6}\right)-i\left(1.18 \times 10^{-8}\right)$ (polymer blend), ${ }^{18} \epsilon_{2}=1-(2.18$ $\left.\times 10^{-5}\right)-i\left(2.63 \times 10^{-6}\right) \quad($ gold $), \quad \epsilon_{3}=1-\left(3.38 \times 10^{-6}\right)$ $-i\left(1.93 \times 10^{-8}\right)$ (silicon), $d_{1}=1000 \AA$ and $d_{2}=500 \AA$, $\sigma_{0}=\sigma_{1}=\sigma_{2}=0$. The critical angles $\theta_{c}=\sqrt{2 \delta}$ are $\theta_{c}($ polymer $)=1.37, \theta_{c}(\mathrm{Au})=4.67$, and $\theta_{c}(\mathrm{Si})=1.84 \mathrm{mrad}$. For $\theta<1.37 \mathrm{mrad}$ incident $\mathrm{x}$-rays will undergo total external reflection from the polymer layer and an evanescent wave will be present in the layer. For $\theta>\theta_{c}$ (polymer) the incident $\mathrm{x}$-rays penetrate into the polymer layer and get reflected strongly by the Au layer up to $\theta=\theta_{c}(\mathrm{Au})$. At $\theta_{c}$ (polymer) $<\theta<\theta_{c}(\mathrm{Au})$ a part of the $\mathrm{x}$-ray beam, already reflected from the Au layer, is reflected back into the polymer layer from the polymer/air interface. The constructive interference between this beam and the incident beam causes enhancement of field intensity in the polymer layer.

For this system the reflectivity $R(\theta)$ is shown as a function of the grazing angle of incidence $(\theta)$ in Fig. 2. The transmitted field intensity [Eq. (18)] into the polymer layer as a function of $\theta$ is also shown in Fig. 2. Only a part of the reflectivity below the critical angle for Au is shown in Fig. 2 to retain the clarity of the oscillatory features of both the curves. [A reflectivity curve well beyond $\theta_{c}(\mathrm{Au})$ is shown later in Fig. 6 for a thinner polymer layer.] It is easy to notice that wherever the reflectivity curve has minima, the transmission curve has maxima. This is expected. The missing intensity in the reflected beam goes into transmission. The enhancement in transmission occurs due to multiple reflections of the beam within the layer at the polymer surface $(0 / 1$ interface) and the polymer/Au (1/2) interface. The field intensities $[I(z)]$ within the top layer for the angles of incidence $(\theta)$ corresponding to the first, second, third, and fourth minima in reflectivity, i.e., at $\theta_{1}=1.4483, \theta_{2}=1.5729, \theta_{3}$ $=1.7621$, and $\theta_{4}=1.9981 \mathrm{mrad}$, respectively, are shown in Fig. 3. We notice that standing-wave patterns are generated within the layer. This also shows that the intensity is enhanced within the layer, the enhancement being the largest for the first order, which is 25 times. This contains the contribution of the two factors in Eq. (17) - the prefactor and the term in the square bracket. We notice from Fig. 3 that the second, third, and fourth orders have 2, 3, and 4 antinodes, respectively, formed within the layer, with decreasing intensity following the transmission pattern in Fig. 2. The threedimensional (3D) intensity plot $[I(\theta, z)]$, showing both $\theta$ and $z$ dependence, is shown in Fig. 4, which also shows the intensity within the second layer $(\mathrm{Au})$. The angles where first-, second-, third-, and fourth-order resonance enhancements occur are noticed on the $\theta$ axis. If we follow the $z$ $=0$ line along the $\theta$ axis in Fig. 4(a) we notice that the intensity goes to $\approx 4$ at the critical angle $\theta_{c}$ (polymer) as expected and then the intensity becomes an oscillatory function of $\theta$. From Fig. 4(b) we notice that corresponding to the angles where field enhancements occur in the polymer layer there are also small enhancements in the underlying $\mathrm{Au}$ layer. This is easily followed, say at the $z=0$ position, along the $\theta$ axis. It means that a part of the resonance-enhanced intensity in the polymer layer leaks into the Au layer. This 


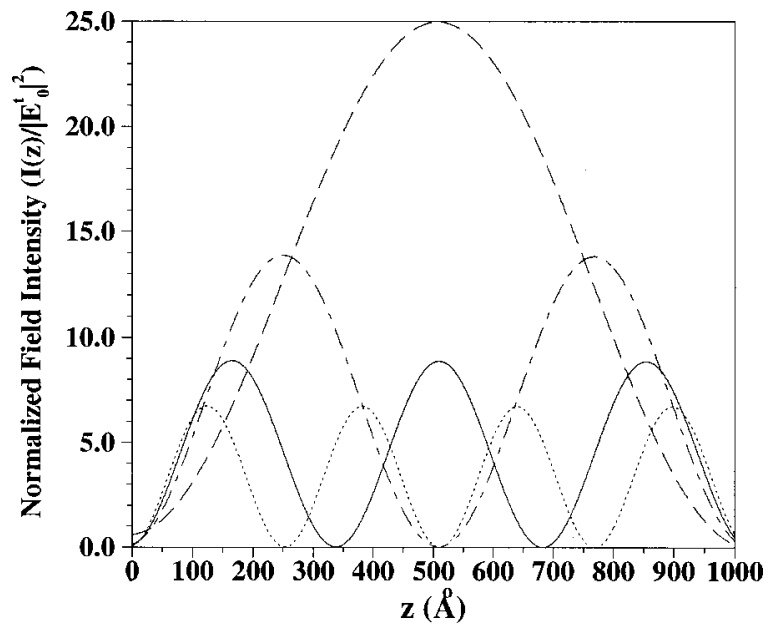

FIG. 3. The field-intensity distribution within the polymer-blend layer for angles of incidence $\theta_{1}=1.4483(---), \theta_{2}=1.5729$ $(---), \theta_{3}=1.7621$ ( -$)$, and $\theta_{4}=1.9981(\ldots \ldots)$ mrad corresponding to first-, second-, third-, and fourth-order minima in reflectivity in Fig. 2. The intensity enhancement is the largest for the first-order with one antinode in the layer [polymer blend(1000 $\mathrm{A})$ / $\mathrm{Au}(500 \AA) / \mathrm{Si}]$.

happens because the polymer/Au interface reflectivity is much less than unity, which is evident in Fig. 2 beyond the polymer critical angle. From Fig. 4(b) we also notice that at the polymer/Au interface $(z=0)$, the intensity in general rises up to $\theta_{c}(\mathrm{Au})$; however, the maximum intensity is much lower than 4 , because of strong (compared to polymer) absorption of $\mathrm{x}$ rays in $\mathrm{Au}$. It is also evident that the penetration of the field into the Au layer increases with $\theta$.

In Fig. 5 we present the field intensity at the middle of the polymer layer, $I(\theta, z=500 \AA)$, and the integrated field intensity, $\int_{0}^{1000 \AA} I(\theta, z) d z$, in the polymer layer. It is clear that in the first case only odd-order intensity enhancement peaks (first, third, fifth, seventh) while in the second case both odd and even orders are observable. This is understood in relation to Fig. 3. For even orders (second, fourth, ... .) there are nodes at $z=500 \AA$. However, the integrated field over the whole depth of the polymer layer is nonzero for all orders. Wang et al. $^{11}$ have demonstrated the first case by using a lipid film with an embedded ultrathin field-intensity sensor layer approximately at the middle of the film. Here we will experimentally demonstrate the second case with the observation of both odd- and even-order enhancements.

The degree of enhancement, the widths of the intensity peaks, and the number of the peaks depend on the layer thickness. This is evident in the plots of Fig. 6 for a $350 \AA$ polymer-blend layer. Figure 6 also shows full curves for angles $(\theta)$ far beyond $\theta_{c}(\mathrm{Au})$. In the experiments we have used such a thin layer. Although the degree of enhancement in this case is less (compare field intensities in Figs. 5 and 6), the requirements on angular precision, beam divergence, etc., are relaxed.

The field intensity can be monitored by detecting a process such as photoelectron yield, Auger-electron yield, fluorescence yield, etc. In our demonstration we will detect fluorescence yield, a process proportional to the field intensity in the dipole approximation, from $\mathrm{Br}$ atoms in the $\mathrm{PBr}_{0.06} \mathrm{~S}$ component in the polymer blend. However, the
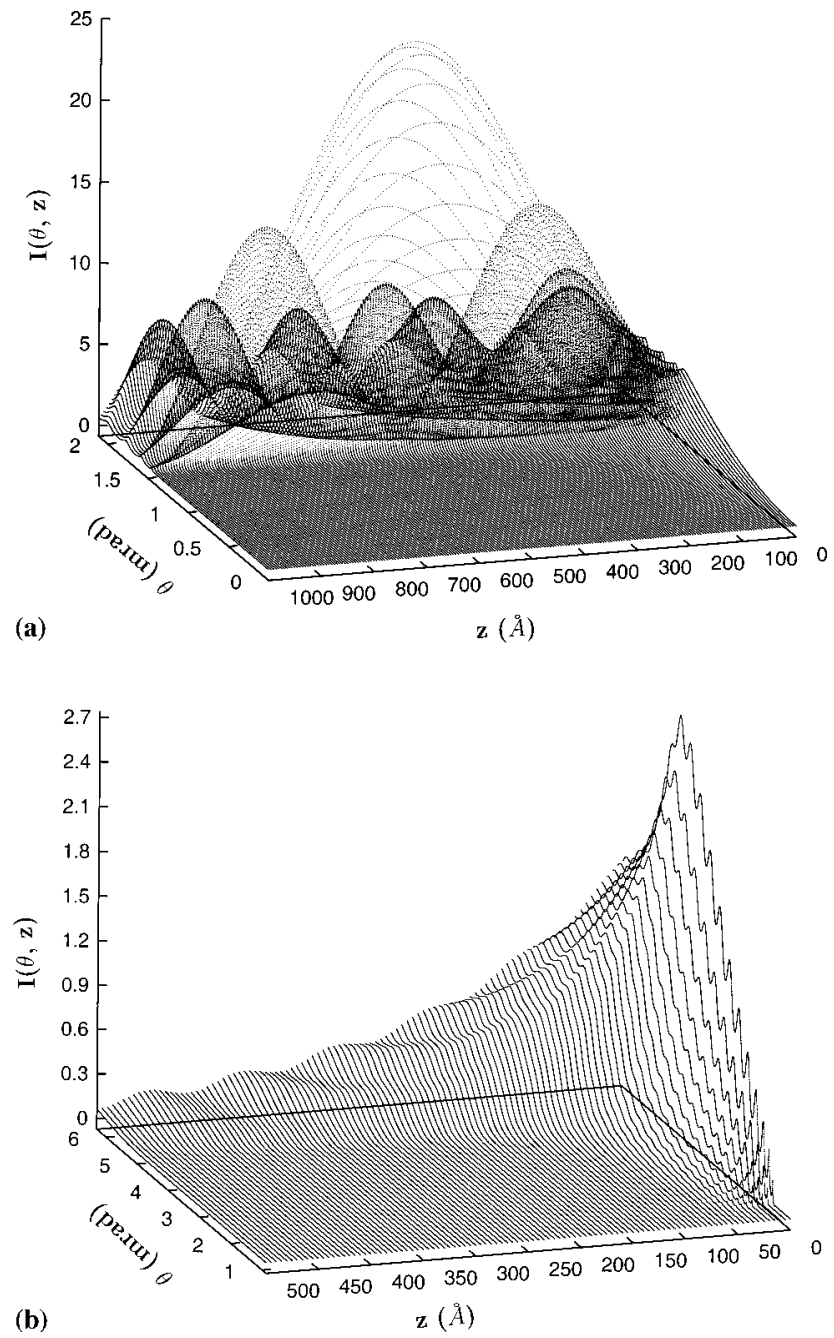

FIG. 4. Three-dimensional intensity plot $I(\theta, z)$ : (a) in the polymer blend, (b) in the Au layer [polymer blend(1000 $\AA) / \mathrm{Au}(500$ $\AA) / \mathrm{Si}]$.

variation of the fluorescence yield with $\theta$ will depend on the distribution of the sensors (here $\mathrm{Br}$ ) within the layer. Two cases are shown in Figs. 5 and 6, where (a) the sensor is concentrated in the middle of the layer and (b) the sensor is

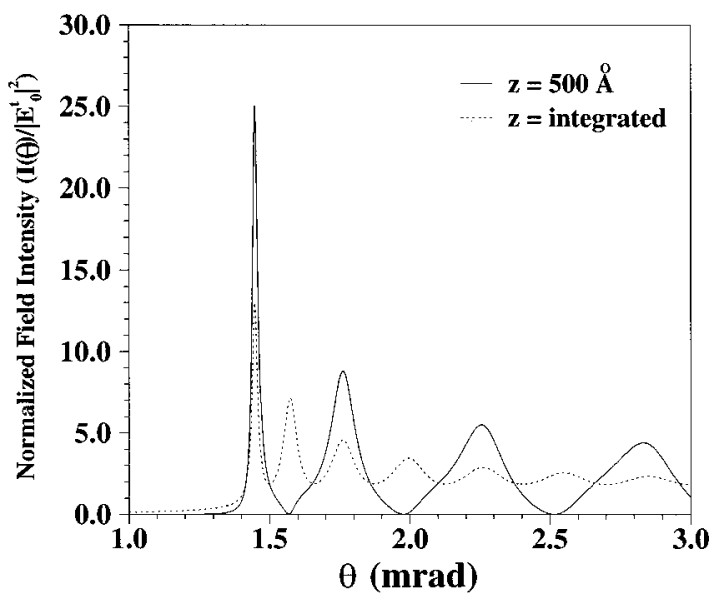

FIG. 5. Field intensity at the middle of the polymer-blend layer $I(\theta, z=500 \AA)$, and the integrated intensity $I(\theta)=\int_{0}^{1000 \AA} I(\theta, z) d z$ [polymer blend $(1000 \AA) / \mathrm{Au}(500 \AA) / \mathrm{Si}$ ]. 


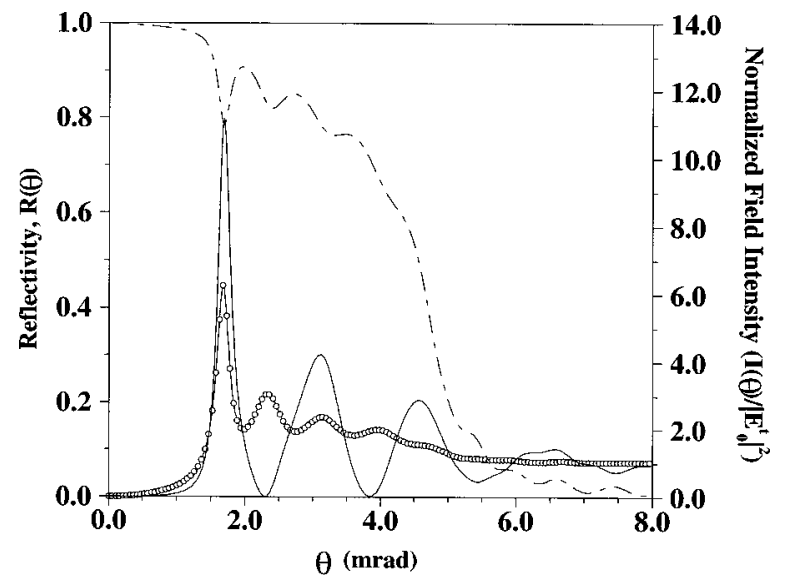

FIG. 6. Theoretical plots of reflectivity (- - -), normalized field intensity at the middle of the polymer blend layer $(z=175 \AA)$ $(-)$, and the integrated field intensity $I(\theta)=\int_{0}^{350 \AA} I(\theta, z) d z$ $\left(-\mathrm{o}^{-}\right)$in the polymer-blend layer [polymer blend $(350 \AA) / \mathrm{Au}(350$ $\AA$ ) $/ \mathrm{Si}]$.

uniformly distributed over the whole thickness of the layer. From the field distribution shown in Fig. 3, the expected fluorescence yield profile from the sensors would be quite different. It is evident from Figs. 5 and 6 if the sensors are uniformly distributed over the depth of the layer both oddand even-order enhancements would be observed. We have a nearly uniform distribution of $\mathrm{PBr}_{x} \mathrm{~S}$ in the polymer-blend layer to observe both odd- and even-order enhancements.

It is clear from Figs. 5 and 6 that the fluorescence yield profile is dependent on the sensor depth distribution. Our second objective is to show that this distribution can be determined from the analysis of the fluorescence yield profile.

\section{APPLICATIONS}

In thin films containing a homogeneous distribution of two components with different surface free energies, the component with lower free energy will tend to segregate towards the free surface. This would lead to an inhomogeneity in the depth distribution of the components. PS and $\mathrm{PBr}_{0.06} \mathrm{~S}$ is a miscible system. A homogeneous mixture of them tend to show a surface enrichment of PS upon annealing. In order to obtain the distribution of one component (here $\mathrm{PBr}_{0.06} \mathrm{~S}$ ) in such a situation one has to obtain the integral field intensity or fluorescence yield. In case the fluorescing atoms are in a distribution given by $f(z)$, the fluorescence yield profile is given by ${ }^{19}$

$$
I_{f}(\theta)=C \int_{0}^{d_{j}} I(\theta, z) f(z) d z,
$$

where $C$ is a normalization constant. The normalized value of $I_{f}(\theta)$ for a uniform distribution $f(z)=$ const of the $\mathrm{PBr}_{x} \mathrm{~S}$ component is equivalent to the integrated field intensity shown in Figs. 5 and 6. $I_{f}(\theta)$ is sensitive to the distribution of the $\mathrm{PBr}_{x} \mathrm{~S}$ component. This gives the possibility to study diffusion, segregation, and spinodal decomposition in polymer-blend systems and interface broadening in layered systems. It has been shown in a theoretical study that with the precision of this method a diffusion coefficient as small as $10^{-21} \mathrm{~cm}^{2} / \mathrm{sec}$ can be measured. ${ }^{20}$ We demonstrate the de-

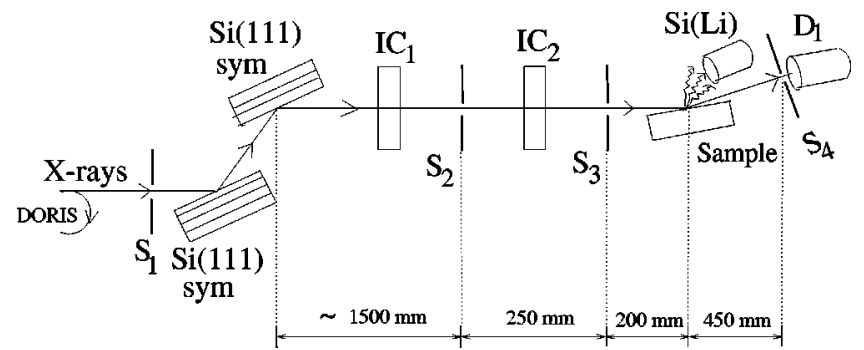

FIG. 7. A schematic view of the experimental setup with a double symmetric $\mathrm{Si}(111)$ crystal monochromator and incident $\mathrm{x}$ rays from the storage ring DORIS. $S_{1}$ (width is $2 \mathrm{~mm}$ ), $S_{2}$ (width is $75 \mu \mathrm{m}$ ), $S_{3}$ (width is $135 \mu \mathrm{m}$ ), $S_{4}$ (width is $75 \mu \mathrm{m}$ ): slits; $I C_{1}$, $I C_{2}$ : ionization chambers; $\mathrm{D} 1$ : diode detector; $\mathrm{Si}(\mathrm{Li})$ : energy dispersive detector.

termination of the depth distribution of the $\mathrm{PBr}_{0.06} \mathrm{~S}$ component in an annealed polymer-blend sample.

\section{EXPERIMENTAL}

A thin film of gold was vacuum evaporated on a silicon wafer substrate $(\mathrm{Au} / \mathrm{Si})$. Subsequently a thin film of a polymer-blend layer (15\% PS, 85\% $\left.\mathrm{PBr}_{0.06} \mathrm{~S}\right)$ was deposited by spin coating on the gold-coated substrate (PS blend / $\mathrm{Au} /$ $\mathrm{Si})$. The sample was annealed at $170^{\circ} \mathrm{C}$ under vacuum $\left(10^{-3}\right.$ Torr) for $10 \mathrm{~h}$. The molecular weight of PS used here is $M_{w}=150 \mathrm{~kg} \mathrm{~mole}{ }^{-1}$ with a narrow molecular weight distribution $\left(M_{w} / M_{n}=1.08\right.$, where $M_{n}$ is the number-average molecular weight). The same PS was used to prepare $\mathrm{PBr}_{0.06} \mathrm{~S}^{17}$

Experiments were performed in HASYLAB at the RÖMO I beam line. The experimental setup is shown in Fig. 7. The scattering geometry is that for $s$ polarization. The diode detector detected the specularly reflected beam and the $\mathrm{Si}(\mathrm{Li})$ detector detected $\mathrm{Br} K \alpha$ fluorescence yield from the $\mathrm{PBr}_{0.06} \mathrm{~S}$ component. The field-intensity enhancement has been sensed through the $\mathrm{Br} K \alpha$ fluorescence yield, a process proportional to the field intensity in the dipole approximation. The average exit angle $\alpha$ [the inclination of the $\mathrm{Si}(\mathrm{Li})$ detector with respect to the sample surface] for fluorescence photons was $25^{\circ}$.

\section{RESULTS AND DISCUSSIONS}

The reflectivity and the $\mathrm{Br} K \alpha$ fluorescence yield are shown in Fig. 8. The lower values of reflected intensity at smaller angles (compared to Fig. 6) are because of a smaller footprint of the incident beam on the sample, where the projection of the beam on the sample is larger than the size of the sample. This footprint effect has been incorporated in the theoretical curve, which also has been convoluted with the beam divergence, and fitted to the experimental data. ${ }^{21} \mathrm{We}$ notice that wherever there is a minimum in the reflectivity, there is a maximum in the fluorescence yield, that is, the field intensity in the polymer layer is maximum corresponding to a minimum in the reflectivity. Here both odd- and even-order enhancement peaks are clearly observed. Earlier only oddorder enhancement peaks have been observed. ${ }^{11}$

The $\mathrm{Br} K \alpha$ fluorescence yield has been analyzed as follows. Although the distribution of the $\mathrm{PBr}_{0.06} \mathrm{~S}$ component in 

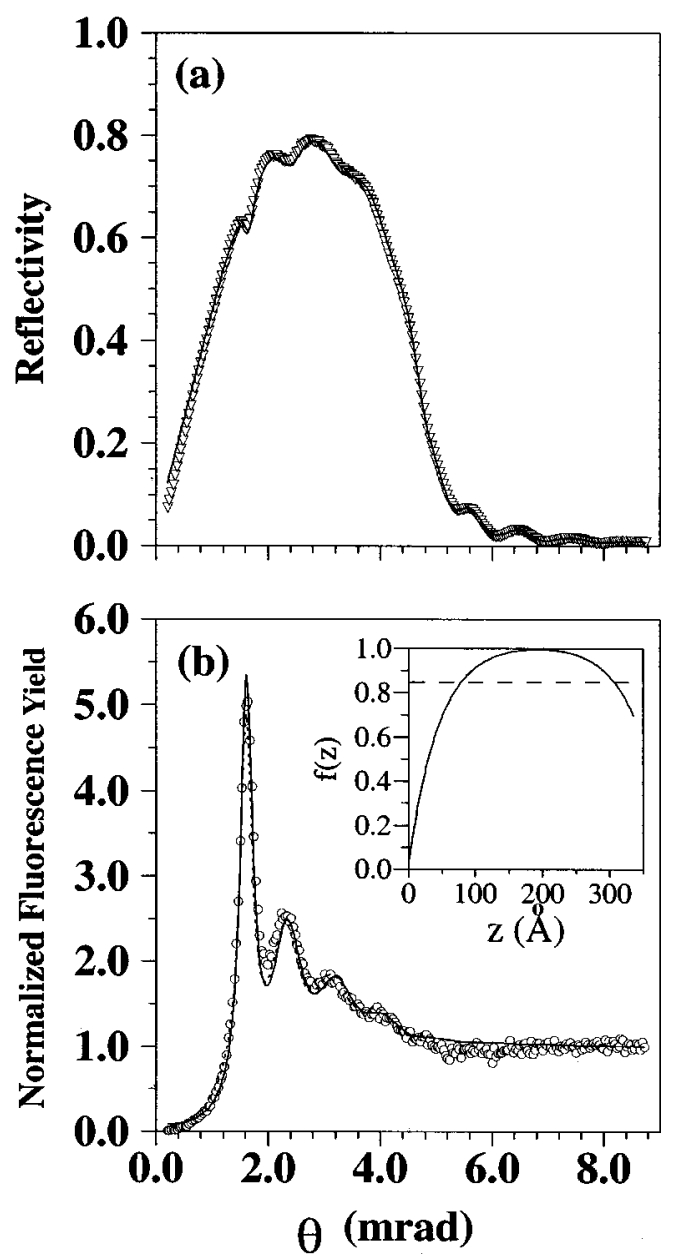

FIG. 8. (a) Reflectivity ( $\nabla$ ) and (b) Br $K \alpha$ fluorescence yield ( $\bigcirc)$ data vs angle of incidence and the fitted curves $(-,----)$ for a polymer-blend layer of PS and $\mathrm{PBr}_{0.06} \mathrm{~S}$ on a Au-coated Si substrate (see text). The solid line in (b) represents a fit according to the surface enrichment model described by Eq. (9). The corresponding $\mathrm{PBr}_{0.06} \mathrm{~S}$ distribution is shown in the inset (solid line). PS is largely enriched at the free surface $(z=0)$ and partially enriched at the blend/Au interface $(z=336 \AA)$. Parameters are given in the text. The dashed lines represent the homogeneous distribution and the corresponding fluorescence fit. The fluorescence yields for both curves have been normalized at $\theta=8.6 \mathrm{mrad}$, where there is no resonance enhancement effect. The $R$ factors for the fits are 0.061 (solid curve) and 0.094 (dashed curve) indicating a better fit for the surface enrichment model. (The first-order peak in the dashed curve is $10 \%$ lower compared to the solid curve.)

the PS- $\mathrm{PBr}_{0.06} \mathrm{~S}$ blend is expected to be homogeneous in the as-prepared sample, from previous neutron reflectivity investigations ${ }^{17}$ one may assume that PS is enriched at the free surface in the annealed sample. Although the enrichment at the layer/substrate interface has not been considered in Ref. 17, one may consider the following general form of the concentration distribution of $\mathrm{PBr}_{0.06} \mathrm{~S}$ over the film thickness:

$$
f(z)=1-\left[\phi_{1} \exp (-z / \xi)+\phi_{2} \exp \left\{-\left(d_{1}-z\right) / \xi\right\}+\phi_{\min }\right] .
$$

This functional form takes into account an enrichment of PS both at the free surface $(z=0)$ and at the layer/substrate

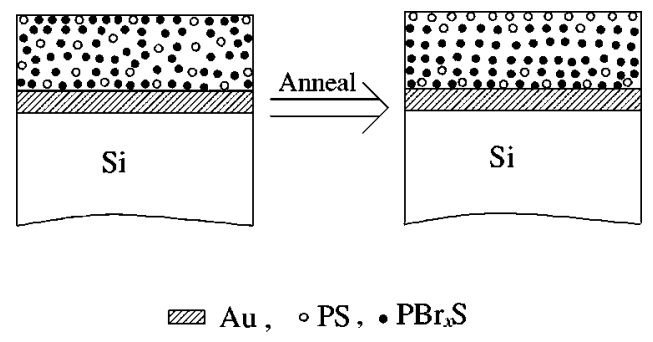

FIG. 9. A schematic diagram showing the initial uniform distribution $\left(85 \% \mathrm{PBr}_{0.06} \mathrm{~S}, 15 \% \mathrm{PS}\right)$ and the distribution after annealing. In the annealed sample, as the distribution in the inset of Fig. 8(b) shows, $\mathrm{PBr}_{0.06} \mathrm{~S}$ is almost completely depleted from the surface and partially depleted from the polymer/Au interface. That is, the surface and the interface are enriched in PS concentration.

interface $\left(z=d_{1}\right)$ with concentration $\phi_{1}$ and $\phi_{2}$, respectively. $z$ is a coordinate perpendicular to the surface and $d_{1}$ is the polymer film thickness. Following theoretical prediction ${ }^{22}$ we use an exponential concentration profile with a decay length $\xi$ discussed previously. ${ }^{8}$ Since $\xi$ should be related to the correlation length for concentration fluctuations in the bulk, ${ }^{22}$ we assume the same decay length at both interfaces. A least-squares fit to the integrated fluorescence yield spectrum, $I_{f}(\theta)$ [Eq. (19)], based on Eq. (20) describes the experimental data better than the best fit with a homogeneous layer [Fig. 8(b)]. Parameters of the fit are as follows: $\phi_{1}=0.99, \phi_{2}=0.32, \phi_{\min }=0, \xi=45 \AA, d_{1}=336 \AA$. (The other parameters for this three-layer system are $d_{2}=282 \AA$, $\sigma_{0}=11 \AA, \sigma_{1}=8 \AA, \sigma_{2}=8 \AA$.) The concentration profile of $\mathrm{PBr}_{0.06} \mathrm{~S}$ is shown in the inset of Fig. 8(b). The calculated reflectivity curves for this model and the homogeneous distribution model are indistinguishable because the electron density of PS and $\mathrm{PBr}_{0.06} \mathrm{~S}$ differ only by $\sim 0.5 \%$. Thus, from simple reflectivity experiments, it is almost impossible to distinguish these models. It is quite evident from the data that (i) PS is highly enriched at the free surface, (ii) PS is slightly enriched at the substrate, and (iii) the fluorescence yield spectrum provides a much clearer evidence of the surface enrichment than the reflectivity data. The enrichment of PS concentration at the polymer surface and at the polymer/Au interface are schematically shown in Fig. 9. The surface enrichment can be understood from energetic considerations taking the surface energies of the components into account. PS has a lower surface tension and surface energy, and is therefore favored at the free surface. The actual surface concentration and decay length are more difficult to predict and may also be influenced by the finite film thickness of the sample and by the second interface, which is close by. Due to this influence the decay length may be smaller than expected from previous experiments. ${ }^{17}$

When the intensity maxima occur in the polymer film, the whole intensity is not confined within the layer. A small part of the field penetrates into the underlying Au layer. This shows as small peaks in the Au $L$-fluorescence yield (not shown here). In principle this contribution can excite additional fluorescence yield in the overlayer. In the present case dominant $\mathrm{Au} L$-fluorescence lines are (in $\mathrm{keV}) L_{\alpha}\left(L_{\alpha 1}\right.$ : 9.7133, $\left.L_{\alpha 2}: 9.6280\right), L_{\beta}\left(L_{\beta 1}: 11.4423, L_{\beta 2}: 11.5847\right)$ and $L_{\gamma}\left(L_{\gamma 1}: 13.3817\right)$. Since the $\operatorname{Br} K$ edge is at $13.474 \mathrm{keV}$, 
these $L$-fluorescence photons from $\mathrm{Au}$ are unable to excite $\mathrm{Br}$ $K$ fluorescence photons, which have been detected and analyzed. Thus the contribution of secondary $\mathrm{Br} K$ fluorescence is absent in this case.

It is clear that the shape of the $\mathrm{Br}$ fluorescence yield curve depends on the depth distribution of $\mathrm{Br}$ or the $\mathrm{PBr}_{0.06} \mathrm{~S}$ component of the polymer blend. In case this component is concentrated around the middle of the film, the even-order enhancement peaks would be weak, while a concentration around a depth of one-fourth of the film thickness would give rise to weaker odd-order peaks. Thus the segregation of this component can be followed. We have observed small peaks on the $\mathrm{Au} L$-fluorescence yield at angles corresponding to reflectivity minima like those shown in Fig. 4(b).

In Sec. II we have discussed the theory for $s$ polarization. In the small $\delta_{j}$ and $\beta_{j}$ approximation, the formulas are the same for both $s$ and $p$ polarization. ${ }^{12}$

\section{CONCLUSIONS}

We have observed both odd- and even-order x-ray resonance enhancement peaks in a polymer-blend layer involving $\mathrm{PS}$ and $\mathrm{PBr}_{x} \mathrm{~S}$. It is clear that the shape of the $\mathrm{Br}$ fluorescence yield curve depends on the depth distribution of $\mathrm{Br}$ or the $\mathrm{PBr}_{x} \mathrm{~S}$ component of the polymer blend. This has been used to extract the distribution of $\mathrm{PBr}_{x} \mathrm{~S}$ in the $\mathrm{PS}-\mathrm{PBr}_{x} \mathrm{~S}$ polymerblend film and to identify a surface and interface enrichment of PS. The decay length, which should be related to the correlation length for concentration fluctuations in the bulk, has been determined. The resonance enhancement phenomenon can be useful in polymer research involving interface broadening, segregation, spinodal decomposition, and diffusion.

For the PS-PBr ${ }_{x} \mathrm{~S}$ blend system miscibility depends on the degree of bromination, $x$. Thus over a range of $x$ the FloryHuggins mean interaction parameter for monomer units of the components can be varied and the diffusion behavior can be studied by this technique. This would help testing current theories.

The application of this technique is not necessarily restricted to polymers. Only the overlayer has to be predominantly materials involving low atomic numbers so that absorption of incident $\mathrm{x}$ rays is small and the critical angle is much smaller compared to the underlying higher atomic number material. Thus it will be possible to study various phenomena in LB films, graphite, fullerine, or even silicon layers.

\section{ACKNOWLEDGMENTS}

This work was supported by the Indo-German bilateral collaboration Project No. Physics-25. B.N.D. was partially supported by HASYLAB. S.D. would like to thank M. H. J. Koch for his support and encouragement.
*FAX: +91-674-581142. Electronic address:

bhupen@iopb.res.in

'Present address: E/1, Institute of Physics Campus, Sachivalaya Marg, Bhubaneswar-751 005 India.

${ }^{1}$ A. H. Compton and S. K. Allison, X-rays in Theory and Experiment (Van Nostrand, New York, 1935).

${ }^{2}$ H. Kiessig, Ann. Phys. (Leipzig) 10, 769 (1931).

${ }^{3}$ P. Eisenberger and W. C. Marra, Phys. Rev. Lett. 46, 1081 (1981).

${ }^{4}$ R. S. Becker, J. A. Golovchenko, and J. R. Patel, Phys. Rev. Lett. 50, 153 (1983).

${ }^{5}$ M. J. Bedzyk, G. M. Bommarito, and J. S. Schildkraut, Phys. Rev. Lett. 62, 1376 (1989).

${ }^{6}$ L. F. Thompson, W. C. Grant, and M. J. Bowden, Introduction to Lithography, 2nd ed. (American Chemical Society, Washington, 1994), p. 308.

${ }^{7}$ D. W. Schubert, Polym. Bull. (Berlin) 38, 177 (1997).

${ }^{8}$ T. P. Russell, Mater. Sci. Rep. 5, 171 (1990), and references therein.

${ }^{9}$ M. Stamm, D.W. Schubert, Annu. Rev. Mater. Sci. 25, 325 (1995).

${ }^{10}$ Y. Feng, S. K. Sinha, H. W. Deckman, J. B. Hastings, and D. P. Siddons, Phys. Rev. Lett. 71, 537 (1993).

${ }^{11}$ J. Wang, M. J. Bedzyk, and M. Caffrey, Science 258, 775 (1992).

${ }^{12}$ D. K. G. de Boer, Phys. Rev. B 44, 498 (1991).

${ }^{13}$ L. G. Parratt, Phys. Rev. 95, 359 (1954).
${ }^{14}$ B. Vidal and P. Vincent, Appl. Opt. 23, 1794 (1984).

${ }^{15}$ B. Pardo, T. Megademini, and J. M. Andre, Rev. Phys. Appl. 23, 1579 (1988).

${ }^{16}$ S. Hüttenbach, M. Stamm, G. Reiter, and M. Foster, Langmuir 7, 2438 (1991).

${ }^{17}$ B. Guckenbiehl, M. Stamm, and T. Springer, Colloids Surf., A 86, 311 (1994).

${ }^{18}$ In order to calculate $\delta$ for the polymer-blend layer we used $\rho(\mathrm{PS})=1.048 \mathrm{gm} / \mathrm{cm}^{3}, \quad \rho\left(\mathrm{PBr}_{x} \mathrm{~S}\right)=(1.048+0.655 x) \mathrm{gm} / \mathrm{cm}^{3}$, and $\rho$ (blend) $=1.08 \mathrm{gm} / \mathrm{cm}^{3}$ (Ref. 17).

${ }^{19}$ Here we have ignored the attenuation of fluorescent photons assuming that the absorption length for the fluorescent photons $\left(\mu_{f}^{-1}\right)$ in the layer is much larger than the layer thickness $d_{j}$, so that $\mu_{f} d_{j} \ll 1$ and $e^{-\mu_{f} d_{j}}$ is $\approx 1$. This essentially means that there is no significant absorption of fluorescent photons on their way to the detector irrespective of the depth $z$ in the layer where they have been produced. In our example, for $\mathrm{Br} K \alpha(11.9 \mathrm{keV})$ fluorescent photons in the polymer-blend layer the linear absorption coefficient is $\mu_{f}=4.807 \mathrm{~cm}^{-1}\left(\mu_{f}^{-1}=2080 \mu \mathrm{m}\right)$ and $d_{j}$ $=350 \AA$, so that $e^{-\mu_{f} d_{j}=0.99998}$. For an average exit angle $\alpha=25^{\circ}$, the factor is $e^{-\mu_{f} d_{j} / \sin \alpha}=0.99996$.

${ }^{20}$ S. Dev and B. N. Dev, Indian J. Phys., A 70, 198 (1996); 70, 543 (1996).

${ }^{21}$ The theoretical curve in Fig. 8(b) has also been corrected for the fact that the fluorescence count increases as $\theta$ increases due to finite aperture of the $\mathrm{Si}(\mathrm{Li})$ detector.

${ }^{22}$ I. Schmidt and K. Binder, J. Phys. (Paris) 46, 1631 (1985). 